BMJ Open

Diabetes

Research

\& Care

\title{
Sex dimorphism in the associations of gestational diabetes with cord blood adiponectin and retinol-binding protein 4
}

\author{
Meng-Nan Yang, ${ }^{1,2}$ Huei-Chen Chiu, ${ }^{2}$ Wen-Juan Wang, ${ }^{3,4}$ Fang Fang, ${ }^{1}$ \\ Guang-Hui Zhang, ${ }^{5}$ Hong Zhu, ${ }^{6}$ Lin Zhang, ${ }^{7}$ Dan-Li Zhang, ${ }^{1}$ Qinwen Du, ${ }^{8}$ Hua He, ${ }^{9}$ \\ Rong Huang, ${ }^{2}$ Xin Liu, ${ }^{1}$ Fei Li, ${ }^{9}$ Jun Zhang, ${ }^{3}$ Fengxiu Ouyang, ${ }^{3}$ Xiaolin Hua,,${ }^{3,10}$ \\ Zhong-Cheng Luo (D) ${ }^{1,2}$
}

To cite: Yang M-N, Chiu H-C, Wang W-J, et al. Sex dimorphism in the associations of gestational diabetes with cord blood adiponectin and retinol-binding protein 4. BMJ Open Diab Res Care 2020;8:e001310. doi:10.1136/ bmjdrc-2020-001310

M-NY and H-CC contributed equally.

Received 24 February 2020

Revised 9 July 2020

Accepted 25 July 2020
Check for updates

(C) Author(s) (or their employer(s)) 2020. Re-use permitted under CC BY-NC. No commercial re-use. See rights and permissions. Published by BMJ.

For numbered affiliations see end of article.

Correspondence to Dr Zhong-Cheng Luo; zcluo@lunenfeld.ca and Dr Xiaolin Hua;

huaxiaolin_sh@163.com

\section{ABSTRACT}

Introduction Gestational diabetes (GD) is associated with impaired insulin sensitivity in newborns. Adiponectin and retinol-binding protein 4 (RBP-4) are involved in regulating insulin sensitivity. Females are more likely to develop diabetes at young ages than males. We tested the hypothesis that GD may affect RBP-4 and adiponectin levels in early life, and there may be sex-dimorphic associations.

Research design and methods In a nested case-control study of 153 matched pairs of neonates of mothers with $\mathrm{GD}$ and euglycemic pregnancies in the Shanghai Birth Cohort, we evaluated cord plasma leptin, high molecular weight (HMW) and total adiponectin and RBP-4 concentrations.

Results Comparing GD versus euglycemic pregnancies adjusted for maternal and neonatal characteristics in female newborns, cord plasma total adiponectin (mean \pm SD: $30.8 \pm 14.3$ vs $37.1 \pm 16.1 \mu \mathrm{g} / \mathrm{mL}, \mathrm{p}=0.048$ ) and HMW adiponectin $(14.6 \pm 7.7$ vs $19.3 \pm 8.3 \mu \mathrm{g} / \mathrm{mL}, \mathrm{p}=0.004)$ concentrations were lower, while RBP-4 concentrations were higher $(21.7 \pm 5.4$ vs $20.0 \pm 4.8 \mu \mathrm{g} / \mathrm{mL}, \mathrm{p}=0.007)$. In contrast, there were no differences in male newborns (all $p>0.2$ ). RBP-4 concentrations were higher in female versus male newborns $(21.7 \pm 5.4$ vs $18.8 \pm 4.5 \mu \mathrm{g} / \mathrm{mL}$, $\mathrm{p}<0.001$ ) in GD pregnancies only. HMW adiponectin concentrations were significantly higher in female versus male newborns in euglycemic pregnancies only $(19.3 \pm 8.3$ vs $16.1 \pm 7.4 \mu \mathrm{g} / \mathrm{mL}, \mathrm{p}=0.014$ ).

Conclusions GD was associated with lower cord plasma HMW adiponectin and higher RBP-4 concentrations in female newborns only. The study is the first to reveal a sex-dimorphic early life impact of GD on metabolic health biomarkers in the offspring. GD may alter the normal presence (HMW adiponectin) or absence (RBP-4) of sex dimorphism in some insulin sensitivity regulation-relevant adipokines in early life.

\section{INTRODUCTION}

Gestational diabetes (GD) is a common metabolic disorder of pregnancy characterized by insulin resistance and altered glucose-lipid metabolism. ${ }^{1}$ The offspring of GD mothers

\section{Significance of this study}

What is already known about this subject?

- Adiponectin and retinol-binding protein 4 (RBP-4) are involved in regulating insulin sensitivity.

- Gestational diabetes is associated with impaired insulin sensitivity in newborns. Gestational diabetes may affect adiponectin and RBP-4 levels in early life, and it is unknown whether there is any sexdimorphic association.

What are the new findings?

- Gestational diabetes is associated with lower cord plasma high molecular weight (HMW) adiponectin and higher RBP-4 concentrations in female newborns but not in male newborns, suggesting a sexdimorphic early life impact on the offspring.

- Gestational diabetes may alter the normal presence (HMW adiponectin) or absence (RBP-4) of sex dimorphism in some insulin sensitivity regulation-relevant adipokines in early life.

How might these results change the focus of research or clinical practice?

- There may be a sex-dimorphic impact of gestational diabetes on metabolic health in the offspring in early life. Further studies are required to understand the long-term metabolic health implications that may inform the development of sex-specific early life interventions to decrease the vulnerability to diabetes.

are at elevated risk of obesity and impaired glucose tolerance during adolescence, and increased risk of diabetes in later life. ${ }^{23}$ How this increased susceptibility to metabolic dysfunctional disorders is developed in early life remains unclear. Impaired insulin sensitivity has been observed in the newborns of mothers with GD, ${ }^{4}$ and in large for gestational age (often a suspected consequence of varying degrees of hyperglycemia in pregnancy) 
newborns, ${ }^{5}$ Plausibly, altered levels of insulin sensitivity regulation adipokines may be involved.

Leptin and adiponectin are important adipokines in regulating insulin sensitivity. ${ }^{6}$ Adiponectin exists in low, medium and high molecular weight (HMW) forms, and HMW adiponectin is recognized as the most bioactive form of adiponectin as related to its insulin-sensitizing property. ${ }^{6}$ Studies have reported elevated cord blood leptin and decreased adiponectin levels in $\mathrm{GD}^{7-9}$ but no significant differences have also been observed in some studies. ${ }^{10}$ Retinol-binding protein 4 (RBP-4) is an adipokine implicated in insulin resistance. ${ }^{11}$ Circulating RBP-4 concentration was positively correlated with insulin resistance in subjects with obesity and type 2 diabetes. ${ }^{12}$ There have been inconsistent data concerning whether there are significant changes in maternal circulating RBP-4 levels in GD. ${ }^{13-17}$ We are aware of only one study on cord blood RBP-4 levels in GD pregnancies. ${ }^{9}$

Studies have demonstrated that females are more insulin resistant than males in utero, ${ }^{18} 19$ and more likely to develop diabetes at young ages. ${ }^{2021}$ It is unclear whether the early life origins of such elevated vulnerability in females may be related to any alterations in insulin sensitivity regulating adipokines such as leptin, adiponectin and RBP-4. Sex dimorphisms in cord blood leptin and adiponectin concentrations have been reported, possibly attributable to the effects of body fat. ${ }^{722}$ It is unknown whether GD may affect any sex dimorphism in fetal (cord) blood levels of leptin, adiponectin and RBP-4.

In view of the above-described knowledge gaps, the present study sought to determine whether there are alterations in cord blood leptin, adiponectin and RBP-4 levels in GD, and assess whether there is sex dimorphism in the associations of GD with cord blood RBP-4, leptin and adiponectin levels in a large birth cohort.

\section{RESEARCH DESIGN AND METHODS}

\section{Study design, subjects and specimens}

This study was based on the recently described Shanghai Birth Cohort (SBC). ${ }^{23}$ The SBC is a large, carefully phenotyped pregnancy/birth cohort with linked biospecimen bank for studies on perinatal determinants of infant growth, development and health. Women at preconception or early pregnancy care clinics were recruited from six urban university-affiliated tertiary obstetric care hospitals in Shanghai between 2013 and 2016 and including a total of 4127 pregnancies. The women were followed up at the first, second and third trimesters of pregnancy and delivery. Data and specimens were collected at each study visit. All collected blood samples were kept on ice, stored temporarily in a $4^{\circ} \mathrm{C}$ refrigerator and centrifuged within 2 hours after the specimen collection. The separated serum and EDTA plasma samples were stored in multiple aliquots at $-80^{\circ} \mathrm{C}$ until assays.

Maternal pre-pregnancy body mass index (BMI, in $\mathrm{kg}$ / $\mathrm{m}^{2}$ ) was calculated as pre-pregnancy weight (self-reported) divided by squared height (measured in the first prenatal care visit). GD was diagnosed according to the International Association of Diabetes and Pregnancy Study Groups criteria ${ }^{24}$-if any one of the blood glucose values was at or above the following thresholds in the $75 \mathrm{~g}$ oral glucose tolerance test at 24-28 weeks of gestation: fasting $5.1 \mathrm{mmol} / \mathrm{L}, 1$-hour $10.0 \mathrm{mmol} / \mathrm{L}$ and 2-hour $8.5 \mathrm{mmol} / \mathrm{L}$.

As part of the SBC project, we conducted a nested casecontrol study on cord blood biomarkers in GD. ${ }^{25}$ The present study reported the original data on the impacts of GD and fetal sex on cord blood leptin, adiponectin and RBP-4 levels (a priori planned study objective). Cases were the newborns of GD mothers, and controls were the newborns of euglycemic mothers. We did not have the data on whether GD mothers were hyperglycemic at the time of delivery (cord blood specimen collection). All cases and controls must meet the following eligibility criteria: (1) Han ethnicity (the majority ethnic group, $>98 \%$ ); (2) maternal age 20-45 years; (3) natural conception; (4) singleton pregnancy; (5) the mother was free of severe pre-pregnancy illnesses (eg, type 1 or type 2 diabetes, and chronic hypertension) and life-threatening pregnancy complications (eg, pre-eclampsia/eclampsia); (6) the infant was free of birth defects; (7) 5 min Apgar score $\geq 7$; and (8) cord blood and placenta specimens available for future assays. All eligible newborns of GD mothers $(n=153)$ were included. Controls were randomly sampled among all eligible subjects and matched to cases (1:1) by infant sex (the same) and gestational age (within 1 week). Therefore, a total of 306 subjects (153 GD; 153 controls) constituted the study sample.

\section{Biochemical assays}

Cord plasma RBP-4 was measured by an ELISA kit (R\&D Systems, Minnesota, USA), and the absorbance was determined using a microplate spectrophotometer (Beckman CX7, USA). Plasma HMW and total adiponectin were measured by an ELISA kit from ALPCO (Salem, NH, USA), and plasma leptin by an ELISA kit from Invitrogen (Carlsbad, CA, USA), respectively. The detection limits were $0.22 \mathrm{ng} / \mathrm{mL}$ for RBP-4, $0.034 \mathrm{ng} / \mathrm{mL}$ for HMW and total adiponectin, and $3.5 \mathrm{pg} / \mathrm{mL}$ for leptin, respectively. Intra-assay and interassay coefficients of variation were in the ranges of $5.0 \%-8.6 \%$ for RBP- 4 , and $6.9 \%-10.4 \%$ for leptin, HMW and total adiponectin, respectively. In all biomarker assays, the laboratory technicians were blinded to the clinical status (GD or not) of study subjects.

\section{Statistical analysis}

The primary outcomes were cord plasma leptin, HMW adiponectin and RBP-4 concentrations. Paired t-test was used to compare continuous variables in GD and control groups, and McNemar's $\chi^{2}$ test was used for comparisons of dichotomous variables. Pearson partial correlation analysis was used to evaluate the correlations between biomarkers adjusting for gestational age at delivery/ blood sampling. Log-transformed biomarker data were used in t-tests, correlation and regression analyses. 
Generalized linear models were applied to assess the differences in cord blood RBP-4, leptin and adiponectin concentrations (by GD status or fetal sex) adjusted for maternal and neonatal characteristics, and in tests for interactions (as multiplicative terms). Maternal and neonatal characteristic variables included maternal age, ethnicity (all Chinese Han), parity, education (university: yes/no), pre-pregnancy BMI $\left(\mathrm{kg} / \mathrm{m}^{2}\right)$, gestational weight gain (z score), smoking or alcohol use in pregnancy (yes/no), GD, gestational hypertension, family history of diabetes, family history of hypertension, mode of delivery (cesarean section/vaginal), infant sex, gestational age (weeks) and birth weight (z score). Parsimonious final regression models were fitted in evaluating the adjusted effects excluding covariables that were not associated with the outcomes ( $>>0.2)$ and did not affect the comparisons. Data management and analyses were performed in SAS V.9.4 (SAS Institute). Two-tailed p value $<0.017$ was considered statistically significant in testing the hypothesis concerning the differences in the three primary outcomes comparing GD versus control groups (Bonferroni correction for three comparisons). A relatively lenient $p$ value $<0.10$ was considered significant in regression tests of interactions to allow better chance of identifying potentially important interactions.

\section{RESULTS}

\section{Maternal and neonatal characteristics}

Characteristics of the study subjects were described recently. ${ }^{25}$ Briefly, women with GD had higher prepregnancy BMI than euglycemic women (mean \pm SD: $23.6 \pm 4.3 \mathrm{vs} 21.6 \pm 2.9 \mathrm{~kg} / \mathrm{m}^{2}, \mathrm{p}<0.001$ ), and were more likely to have gestational hypertension $(5.2 \%$ vs $0.6 \%, \mathrm{p}=0.036)$, and tended to be more likely to have a family history of diabetes $(16.5 \%$ vs $9.5 \%, \mathrm{p}=0.072)$. There were no significant differences in other maternal characteristics including maternal age (30.4 \pm 3.7 vs $29.9 \pm 3.4$ years), education (university $62.8 \%$ vs $60.5 \%$ ), parity (primiparous $81.1 \%$ vs $81.7 \%)$, alcohol use $(7.1 \%$ vs $11.3 \%$ ) and smoking during pregnancy (only three smokers) (all p $>0.1$ ). Newborns of GD mothers were more likely to be delivered by cesarean section than newborns of euglycemic mothers (54.3\% vs $33.1 \%, \mathrm{p}<0.001)$. Despite the matching for infant sex and gestational age at delivery (within 1 week), mean gestational age at delivery was slightly shorter comparing GD versus euglycemic pregnancies $(39.1 \pm 1.2$ vs $39.3 \pm 1.2$ weeks, $\mathrm{p}<0.001)$, while average birth weight tended to be higher ( $3435.9 \pm 488.7$ vs $3354.6 \pm 435.6 \mathrm{~g}, \mathrm{p}=0.093)$.

\section{Cord plasma RBP-4, leptin and adiponectin in GD and euglycemic pregnancies}

Adjusting for maternal and neonatal characteristics including pre-pregnancy BMI, family history of hypertension, gestational hypertension, family history of diabetes, cesarean section, and gestational age at delivery (other covariates did not affect the comparisons), cord plasma HMW adiponectin concentrations and HMW/ total adiponectin ratios were lower in GD versus euglycemic pregnancies in the total study sample (table 1). However, there were no significant differences in cord plasma RBP-4, total adiponectin and leptin concentrations between the two groups.

\section{Sex-specific associations of GD with RBP-4, leptin and adiponectin}

Adjusted for maternal and neonatal characteristics, there were no significant differences in all observed cord blood

Table 1 Cord plasma RBP-4, leptin, HMW and total adiponectin concentrations in the newborns of GD versus control (euglycemic) mothers

\begin{tabular}{|c|c|c|c|c|}
\hline & GD (n=153) & Control $(n=153)$ & $\begin{array}{l}\text { Crude } \\
\text { P value* }\end{array}$ & $\begin{array}{l}\text { Adjusteo } \\
\text { P value* }\end{array}$ \\
\hline \multirow[t]{2}{*}{ RBP-4 ( $\mu \mathrm{g} / \mathrm{mL})$} & $20.1 \pm 5.1$ & $19.7 \pm 5.0$ & 0.562 & 0.204 \\
\hline & $19.5(16.8,23.2)$ & $19.0(15.9,22.7)$ & & \\
\hline \multirow[t]{2}{*}{ Leptin (ng/mL) } & $7.3 \pm 7.3$ & $8.1 \pm 7.1$ & 0.074 & 0.341 \\
\hline & $4.6(2.8,9.4)$ & $5.9(3.6,9.9)$ & & \\
\hline \multirow[t]{2}{*}{ Adiponectin, $\mathrm{HMW}(\mu \mathrm{g} / \mathrm{mL})$} & $15.0 \pm 7.5$ & $17.6 \pm 8.0$ & 0.002 & 0.006 \\
\hline & $14.3(10.1,18.8)$ & $16.3(12.1,21.6)$ & & \\
\hline \multirow[t]{2}{*}{ Adiponectin, total $(\mu \mathrm{g} / \mathrm{mL})$} & $33.2 \pm 15.0$ & $36.3 \pm 15.8$ & 0.073 & 0.071 \\
\hline & $31.7(23.5,42.6)$ & $33.1(24.7,46.1)$ & & \\
\hline \multirow[t]{2}{*}{ HMW/total ratio } & $0.46 \pm 0.15$ & $0.50 \pm 0.14$ & 0.003 & 0.032 \\
\hline & $0.44(0.36,0.51)$ & $0.49(0.41,0.56)$ & & \\
\hline
\end{tabular}

Data presented are mean \pm SD and median (IQR).

$P$ values in bold: $p<0.017$ (significant after accounting for multiple tests of the differences in the three primary outcomes (leptin, HMW adiponectin and RBP-4) between GD and control groups).

${ }^{*}$ Crude $p$ values were from paired t-tests in log-transformed data. Adjusted $p$ values were from generalized linear models in the comparisons of log-transformed biomarker data between the two groups adjusted for maternal (pre-pregnancy BMl, family history of diabetes, family history of hypertension, gestational hypertension) and neonatal (cesarean section) characteristics; other factors were excluded since they were similar and did not affect the comparisons $(p>0.2)$.

BMI, body mass index; GD, gestational diabetes; HMW, high molecular weight; RBP-4, retinol-binding protein 4. 
Table 2 Cord plasma RBP-4, leptin and adiponectin concentrations in the newborns of GD versus control (euglycemic) mothers stratified by infant sex

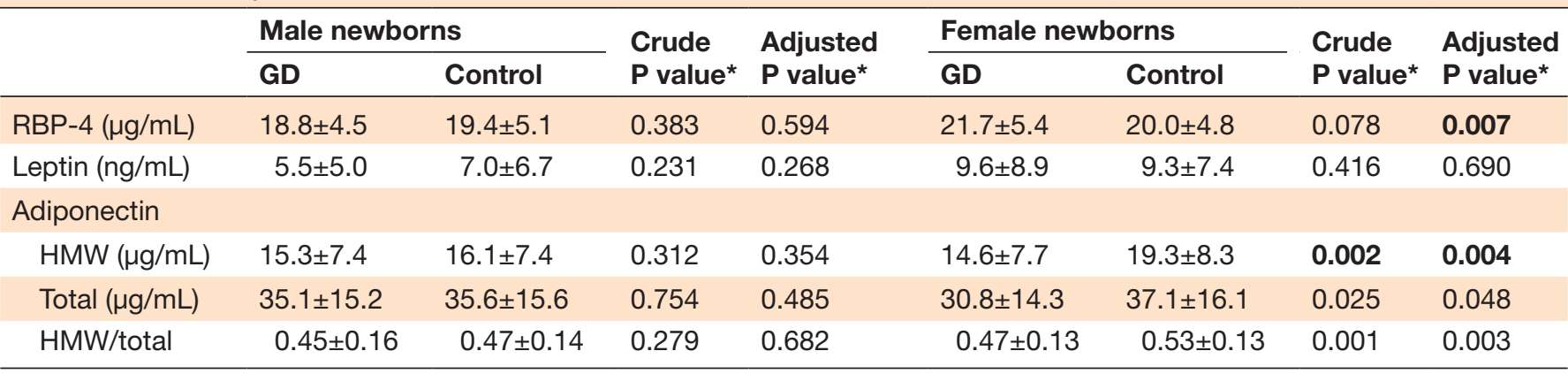

Data presented are mean \pm SD. There were 140 male newborns (of $70 \mathrm{GD}$ and 70 euglycemic mothers) and 166 female newborns (of 83 GD and 83 euglycemic mothers) in the analyses.

$P$ values in bold: $p<0.017$ (significant after accounting for multiple tests of the differences in the three primary outcomes (leptin, HMW adiponectin and RBP-4) between GD and control groups).

${ }^{*}$ Crude $p$ values were from paired t-tests in log-transformed data. Adjusted $p$ values were from generalized linear models in the comparisons of log-transformed biomarker data between the two groups adjusted for maternal (pre-pregnancy BMI, family history of diabetes, family history of hypertension, gestational hypertension) and neonatal (cesarean section) characteristics; other factors were excluded since they were similar and did not affect the comparisons $(p>0.2)$.

BMI, body mass index; GD, gestational diabetes; HMW, high molecular weight; RBP-4, retinol-binding protein 4.

biomarkers between GD and non-GD groups in male newborns (table 2). In contrast, for female newborns, GD was associated with higher cord plasma RBP-4 concentrations (adjusted $\mathrm{p}=0.007$ ), but lower HMW adiponectin (adjusted $\mathrm{p}=0.004$ ) and total adiponectin (adjusted $\mathrm{p}=0.048$, not significant if accounting for multiple tests) concentrations, and lower HMW/total adiponectin ratios. The $\mathrm{p}$ values in tests for interactions of GD with infant sex were 0.094 for RBP-4, 0.035 for HMW adiponectin, 0.114 for total adiponectin, and 0.198 for HMW/total adiponectin ratio, respectively. The adjusted differences (in $\log$ scale) are presented in table 3 , and the findings were similar as in table 2.

\section{Sex differences in cord blood RBP-4, leptin and adiponectin}

In GD pregnancies, female newborns had significantly higher cord plasma RBP-4 concentrations than male newborns $(21.7 \pm 5.4$ vs $18.8 \pm 4.5 \mathrm{ng} / \mathrm{mL}, \mathrm{p}<0.001)$, while there was no sex difference in euglycemic pregnancies $(p=0.43)$. In euglycemic pregnancies, there were higher HMW adiponectin concentrations $(\mathrm{p}=0.014)$ and HMW/ total adiponectin ratios $(\mathrm{p}=0.006)$ in female versus male newborns, while these differences disappeared in GD pregnancies $(\mathrm{p}=0.59$ for HMW adiponectin; $\mathrm{p}=0.47$ for HMW/total adiponectin ratio). Cord plasma leptin levels were higher in female versus male newborns regardless of GD status ( $\mathrm{p}=0.001$ in GD; $\mathrm{p}=0.045$ in controls).

\section{Partial correlations}

Adjusting for gestational age at blood sampling, cord plasma RBP-4 was weakly and positively correlated to leptin and birth weight $\mathrm{z}$ score, but not correlated to adiponectin (table 4). As expected, HMW and total adiponectin were strongly correlated. Leptin was

Table 3 The adjusted differences in cord blood biomarkers between GD and controls in male and female newborns

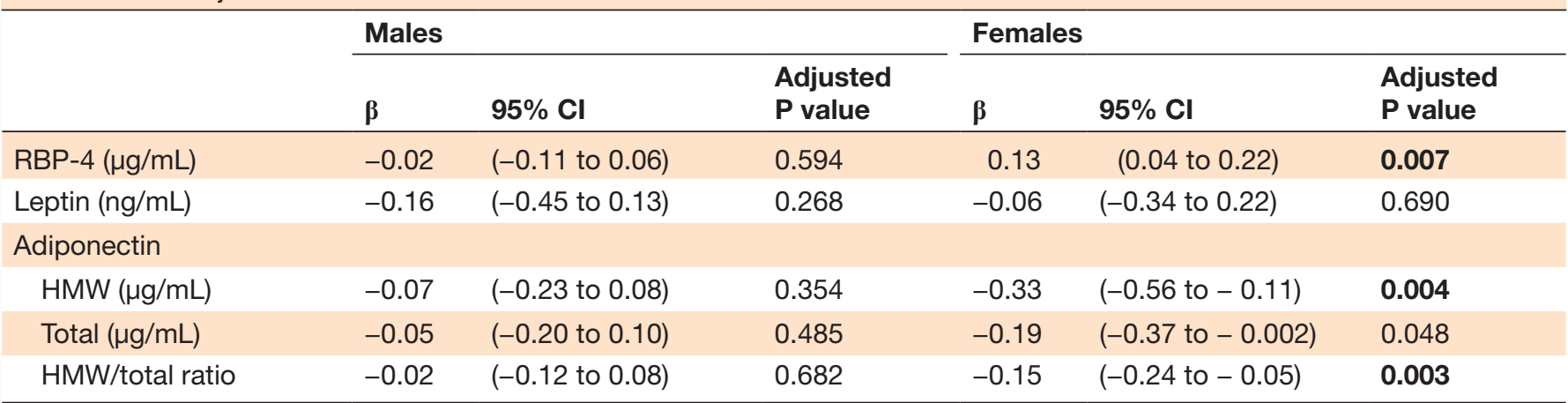

Data ( $\beta$ ) presented are the regression coefficients for GD (yes vs no) from generalized linear models of log-transformed biomarker data with the adjustments for maternal (pre-pregnancy BMI, family history of diabetes, family history of hypertension, gestational hypertension) and neonatal (cesarean section) characteristics; other factors were excluded since they were similar and did not affect the comparisons between the two groups.

$P$ values in bold: $p<0.017$ (significant after accounting for multiple tests of the differences in the three primary outcomes (leptin, HMW adiponectin, RBP-4) between GD and controls).

BMI, body mass index; GD, gestational diabetes; HMW, high molecular weight; RBP-4, retinol-binding protein 4. 
Table 4 Partial correlation coefficients ( $r$ ) between cord blood RBP-4, leptin, HMW and total adiponectin and birth weight $z$ score

\begin{tabular}{lllll}
\hline & RBP-4 & Leptin & HMW adiponectin & Total adiponectin \\
\hline Leptin & $0.13^{\star}$ & & & \\
Adiponectin, HMW & 0.07 & $0.18^{\star \star}$ & & \\
Adiponectin, total & 0.05 & 0.09 & $0.85^{\star \star \star}$ & $0.15^{\star}$ \\
Birth weight, z score & $0.15^{\star \star}$ & $0.37^{\star \star \star}$ & $0.12^{\star}$ & \\
\hline
\end{tabular}

Data presented are Pearson partial correlation adjusting for gestational age at delivery/cord blood sampling.

${ }^{*} \mathrm{P}<0.05 ;{ }^{* *} \mathrm{p}<0.01 ;{ }^{* \star *} \mathrm{p}<0.001$.

HMW, high molecular weight; RBP-4, retinol-binding protein 4.

positively correlated to HMW adiponectin. Sex-stratified analyses showed that RBP-4 was positively correlated with leptin in female newborns but not in male newborns (table 5).

\section{DISCUSSION}

\section{Main findings}

The study is the first to reveal sex-dimorphic associations of GD with cord blood RBP-4 and adiponectin concentrations. GD was associated with elevated cord blood RBP-4 and decreased HMW adiponectin concentrations in female newborns but not in male newborns. The study is the first to demonstrate that GD may affect the normal presence (HMW adiponectin) or absence (RBP-4) of sex dimorphism in some insulin sensitivity regulationrelevant adipokines in early life.

\section{Data interpretation and comparisons to findings in previous studies}

Adipokines play a critical role in the regulation of glucose homeostasis and insulin sensitivity, ${ }^{12} 26$ and may provide an important link between obesity-related disorders and insulin resistance. ${ }^{27} 28$ RBP-4 is involved in regulating glucose metabolism and insulin sensitivity. ${ }^{12}{ }^{29}$ Some studies have reported higher maternal serum RBP-4 levels in GD women. ${ }^{9} 1314$ Two studies ${ }^{9} 13$ reported no significant differences in cord serum RBP-4 concentrations between GD and control groups, but they did not report sex-specific comparisons. In contrast, our

Table 5 Correlations of cord plasma RBP-4 with leptin, HMW and total adiponectin in male and female newborns

\begin{tabular}{llllll}
\hline & \multicolumn{2}{l}{ Males } & & \multicolumn{2}{l}{ Females } \\
\cline { 2 - 3 } \cline { 5 - 6 } & $\mathbf{r}$ & P value & & $\mathbf{r}$ & P value \\
\hline Leptin & 0.012 & 0.884 & & 0.2147 & $\mathbf{0 . 0 1 3}$ \\
Adiponectin & & & & \\
$\quad$ HMW & 0.145 & 0.067 & & -0.010 & 0.912 \\
$\quad$ Total & 0.123 & 0.121 & & -0.005 & 0.952 \\
HMW/total ratio & 0.078 & 0.377 & & -0.059 & 0.947 \\
\hline
\end{tabular}

Data presented are Pearson partial correlations ( $r$ ) adjusting for gestational age at delivery/cord blood sampling.

$P$ values in bold: $p<0.05$.

$\mathrm{HMW}$, high molecular weight; RBP-4, retinol-binding protein 4. data showed that GD was associated with elevated cord plasma RBP-4 concentrations in female newborns only, suggesting that GD may have a sex-dimorphic impact on metabolic health during fetal life.

Two studies reported that total or HMW adiponectin concentrations in cord serum did not differ between GD and controls, ${ }^{30} 31$ whereas two other studies reported lower cord serum or plasma adiponectin concentrations in GD. ${ }^{89}$ In contrast, we observed that GD was associated with decreased cord plasma adiponectin (more substantially for HMW adiponectin) concentrations in female newborns, but not in male newborns. Previous studies did not provide sex-specific data. Our data from a nested case-control study in a much larger cohort suggest a sex-dimorphic impact of GD on cord blood adiponectin concentration.

Some studies in adolescents or adults reported sex differences in circulating RBP-4 levels, ${ }^{29} 32$ while others did not. ${ }^{33}$ Serum RBP-4 concentrations were observed to be lower in female than male subjects in children and adults. $^{29} 3435$ There is a lack of data on sex difference in cord blood RBP-4. We observed no sex difference in cord plasma RBP-4 concentrations in euglycemic pregnancies, but higher concentrations in female newborns in GD pregnancies. The mechanisms are unclear. We speculate that this may be due to the altered metabolic milieu in GD with pronounced insulin resistance. ${ }^{36}$

Higher total adiponectin circulating concentrations in females versus males have been reported in children, adolescents and adults. ${ }^{37-39}$ This sex dimorphism appears to be primarily related to the HMW adiponectin complexes. $^{39} 40$ Our data in euglycemic pregnancies suggest that this sex dimorphism in HMW adiponectin may be established in utero in normal (euglycemic) pregnancies (higher cord plasma HMW adiponectin concentrations in female vs male neonates). HMW adiponectin levels may vary by fetal sex even at the early stage of human development. We speculated that this sex dimorphism could be related to the effects of androgens. Testosterone may selectively reduce HMW adiponectin secretion from adipocytes, ${ }^{414}$ and higher testosterone concentrations in male versus female fetuses ${ }^{43}$ may contribute to the lower HMW concentrations in male newborns. Androgens may indirectly regulate adiponectin distribution through a decrease in adipocyte number and size in male fetuses. ${ }^{445}$ 
Importantly, our study is the first to reveal that such physiological sex difference in cord blood HMW adiponectin levels may be lost in GD pregnancies.

\section{Strengths and limitations}

The main strengths include the large birth cohort, timely collection and processing of blood specimens and highquality biochemical assays (low intra-assay and interassay coefficients of variation), and sex-specific data. The main limitation is the observational nature of the study. We could not rule out the possibility of reverse causality. The study was limited to Chinese Han ethnicity subjects. While such study design confers the advantage of enhanced power to uncover biological relationships in the absence of the potential confounding effects of race/ethnicity, it also calls for more studies in other race/ethnic groups to understand the generalizability of the study findings.

\section{CONCLUSIONS}

GD may affect cord blood RBP-4 and adiponectin levels in female newborns but not in male newborns. GD may alter the normal presence (HMW adiponectin) or absence (RBP-4) of sex dimorphism in some insulin sensitivity regulation-relevant adipokines in early life. Further studies are required to understand the longterm metabolic health implications, and physicians may be informed for better counseling of patients, and this knowledge will inform the development of early life sexspecific interventions to decrease the vulnerability to diabetes in future generations.

\section{Author affiliations}

${ }^{1}$ Ministry of Education-Shanghai Key Laboratory of Children's Environmental Health, and Department of Pediatrics, Xinhua Hospital Affiliated to Shanghai Jiaotong University School of Medicine, Shanghai, China

${ }^{2}$ Department of Obstetrics and Gynecology, Lunenfeld-Tanenbaum Research Institute, Prosserman Centre for Population Health Research, Mount Sinai Hospital, Institute of Health Policy, Management and Evaluation, Dalla Lana School of Public Health, Faculty of Medicine, University of Toronto, Toronto, Ontario, Canada ${ }^{3}$ Ministry of Education-Shanghai Key Laboratory of Children's Environmental Health, Xinhua Hospital Affiliated to Shanghai Jiaotong University School of Medicine, Shanghai, China

${ }^{4}$ Department of Obstetrics and Gynecology, Lunenfeld-Tanenbaum Research Institute, Mount Sinai Hospital, University of Toronto, Toronto, Ontario, Canada ${ }^{5}$ Department of Clinical Assay Laboratory, Xinhua Hospital Affiliated to Shanghai Jiaotong University School of Medicine, Shanghai, China

${ }^{6}$ Department of Obstetrics and Gynecology, Xinhua Hospital Affiliated to Shanghai Jiaotong University School of Medicine, Shanghai, China

${ }^{7}$ Obstetrics and Gynecology, International Peace Maternity and Child Health Hospital, Shanghai Jiao-Tong University School of Medicine, Shanghai, China ${ }^{8}$ Department of Obstetrics and Gynecology, Ruijin Hospital Affiliated to Shanghai Jiao Tong University Medical School, Shanghai, China

${ }^{9}$ Department of Pediatrics, Xinhua Hospital Affiliated to Shanghai Jiaotong University School of Medicine, Shanghai, China

${ }^{10}$ Obstetrics and Gynecology, Shanghai First Maternity and Infant Hospital, Tongji University School of Medicine, Shanghai, China

Acknowledgements We gratefully acknowledged all research staff who had contributed to patient recruitment and data collection in the Shanghai Birth Cohort.

Contributors ZCL, HZ, GHZ, LZ, FL, JZ, FO and XH conceived the study with inputs from all coauthors. MNY, WJW, FF, GHZ, HZ, LZ, DLZ, QD, HH, RH, XL, FL, JZ, FO and ZCL contributed to the acquisition of research data. WJW and HCC conducted the literature review and data analysis and drafted the manuscript. All authors contributed in revising the article critically for important intellectual content, and approved the final version for publication.

Funding This work was supported by research grants from the Ministry of Science and Technology of China (2019YFA0802501), the National Natural Science Foundation of China (81673178, 81903323, 81761128035 and 81930095), the Shanghai Municipal Health Commission (2020CXJQ01 and 201640032), the Canadian Institutes of Health Research (158616), the Shanghai Science and Technology Commission (19410713500), and the National Human Genetic Resources Sharing Service Platform (2005DKA21300).

Competing interests None declared.

\section{Patient consent for publication Not required.}

Ethics approval The study was approved by the Research Ethics Committees of the coordination center (Shanghai Xinhua Hospital, reference number M2013-010) and all participating hospitals. Written informed consent was obtained from all study participants.

Provenance and peer review Not commissioned; externally peer reviewed.

Data availability statement Data are available upon reasonable request. Access to the deidentified participant research data must be approved by the research ethics board on a case-by-case basis; please contact the corresponding author (zcluo@lunenfeld.ca; huaxiaolin_sh@163.com) for assistance in data access request.

Open access This is an open access article distributed in accordance with the Creative Commons Attribution Non Commercial (CC BY-NC 4.0) license, which permits others to distribute, remix, adapt, build upon this work non-commercially, and license their derivative works on different terms, provided the original work is properly cited, appropriate credit is given, any changes made indicated, and the use is non-commercial. See: http://creativecommons.org/licenses/by-nc/4.0/.

ORCID iD

Zhong-Cheng Luo http://orcid.org/0000-0002-1794-1312

\section{REFERENCES}

1 Waters TP, Kim SY, Sharma AJ, et al. Longitudinal changes in glucose metabolism in women with gestational diabetes, from late pregnancy to the postpartum period. Diabetologia 2020;63:385-94.

2 Reece EA. The fetal and maternal consequences of gestational diabetes mellitus. J Matern Fetal Neonatal Med 2010;23:199-203.

3 Page KA, Luo S, Wang X, et al. Children exposed to maternal obesity or gestational diabetes mellitus during early fetal development have hypothalamic alterations that predict future weight gain. Diabetes Care 2019;42:1473-80.

4 Luo Z-C, Delvin E, Fraser WD, et al. Maternal glucose tolerance in pregnancy affects fetal insulin sensitivity. Diabetes Care 2010;33:2055-61.

5 Dong Y, Luo Z-C, Nuyt AM, et al. Large-for-Gestational-Age may be associated with lower fetal insulin sensitivity and $\beta$-cell function linked to leptin. J Clin Endocrinol Metab 2018;103:3837-44.

6 Zhang $\mathrm{H}$, Zhang $\mathrm{C}$. Adipose "talks" to distant organs to regulate insulin sensitivity and vascular function. Obesity 2010;18:2071-6.

7 Okereke NC, Uvena-Celebrezze J, Hutson-Presley L, et al. The effect of gender and gestational diabetes mellitus on cord leptin concentration. Am J Obstet Gynecol 2002;187:798-803.

8 Cortelazzi D, Corbetta S, Ronzoni S, et al. Maternal and foetal resistin and adiponectin concentrations in normal and complicated pregnancies. Clin Endocrinol 2007;66:447-53.

9 Ortega-Senovilla H, Schaefer-Graf U, Meitzner K, et al. Gestational diabetes mellitus causes changes in the concentrations of adipocyte fatty acid-binding protein and other adipocytokines in cord blood. Diabetes Care 2011;34:2061-6.

10 Luo Z-C, Nuyt A-M, Delvin E, et al. Maternal and fetal leptin, adiponectin levels and associations with fetal insulin sensitivity. Obesity 2013;21:210-6.

11 Tsutsumi C, Okuno M, Tannous L, et al. Retinoids and retinoidbinding protein expression in rat adipocytes. $J$ Biol Chem 1992;267:1805-10.

12 Yang Q, Graham TE, Mody N, et al. Serum retinol binding protein 4 contributes to insulin resistance in obesity and type 2 diabetes. Nature 2005;436:356-62.

13 Chan T-F, Chen H-S, Chen Y-C, et al. Increased serum retinolbinding protein 4 concentrations in women with gestational diabetes mellitus. Reprod Sci 2007;14:169-74. 
14 Su Y-X, Hong J, Yan Q, et al. Increased serum retinol-binding protein-4 levels in pregnant women with and without gestational diabetes mellitus. Diabetes Metab 2010;36:470-5.

15 Kuzmicki M, Telejko B, Wawrusiewicz-Kurylonek N, et al. RetinolBinding protein 4 in adipose and placental tissue of women with gestational diabetes. Gynecol Endocrinol 2011;27:1065-9.

16 Abetew DF, Qiu C, Fida NG, et al. Association of retinol binding protein 4 with risk of gestational diabetes. Diabetes Res Clin Pract 2013;99:48-53.

17 Maghbooli Z, Hossein-nezhad A, Mirzaei K, et al. Association between retinol-binding protein 4 concentrations and gestational diabetes mellitus and risk of developing metabolic syndrome after pregnancy. Reprod Sci 2010;17:196-201.

18 Shields BM, Knight B, Hopper H, et al. Measurement of cord insulin and insulin-related peptides suggests that girls are more insulin resistant than boys at birth. Diabetes Care 2007;30:2661-6.

19 Mittendorfer B. Insulin resistance: sex matters. Curr Opin Clin Nutr Metab Care 2005;8:367-72.

20 Grinstein G, Muzumdar R, Aponte L, et al. Presentation and 5-year follow-up of type 2 diabetes mellitus in African-American and Caribbean-Hispanic adolescents. Horm Res Paediatr 2003;60:121-6.

21 Wei J-N, Sung F-C, Lin C-C, et al. National surveillance for type 2 diabetes mellitus in Taiwanese children. JAMA 2003;290:1345-50.

22 Basu S, Laffineuse L, Presley L, et al. In utero gender dimorphism of adiponectin reflects insulin sensitivity and adiposity of the fetus. Obesity 2009;270:1144-9.

23 Zhang J, Tian Y, Wang W, et al. Cohort profile: the Shanghai birth cohort. Int J Epidemiol 2019;48:21-21g.

24 Metzger BE, Gabbe SG, Persson B, et al. International association of diabetes and pregnancy study groups recommendations on the diagnosis and classification of hyperglycemia in pregnancy: response to Weinert. Diabetes Care 2010;33:e98.

25 Wang W-J, Zhang L, Zheng T, et al. Fetuin-A and fetal growth in gestational diabetes mellitus. BMJ Open Diabetes Res Care 2020;8:e000864.

26 Graham TE, Yang Q, Blüher $\mathrm{M}$, et al. Retinol-binding protein 4 and insulin resistance in lean, obese, and diabetic subjects. $N$ Engl $J$ Med 2006;354:2552-63.

27 Shepherd PR, Gnudi L, Tozzo E, et al. Adipose cell hyperplasia and enhanced glucose disposal in transgenic mice overexpressing GLUT4 selectively in adipose tissue. J Biol Chem 1993;268:22243-6.

28 Abel ED, Peroni O, Kim JK, et al. Adipose-selective targeting of the GLUT4 gene impairs insulin action in muscle and liver. Nature 2001;409:729-33.

29 Cho YM, Youn B-S, Lee H, et al. Plasma retinol-binding protein-4 concentrations are elevated in human subjects with impaired glucose tolerance and type 2 diabetes. Diabetes Care 2006;29:2457-61.

30 Uebel K, Pusch K, Gedrich K, et al. Effect of maternal obesity with and without gestational diabetes on offspring subcutaneous and preperitoneal adipose tissue development from birth up to year-1. BMC Pregnancy Childbirth 2014;14:138
31 Ballesteros M, Simon I, Vendrell J, et al. Maternal and cord blood adiponectin multimeric forms in gestational diabetes mellitus: a prospective analysis. Diabetes Care 2011;34:2418-23.

32 Lin C-J, Chu NF, Hung Y-J, et al. The association of retinol-binding protein 4 with metabolic syndrome and obesity in adolescents: the effects of gender and sex hormones. Clin Pediatr 2013;52:16-23.

33 Kos K, Wong S, Tan BK, et al. Human RBP4 adipose tissue expression is gender specific and influenced by leptin. Clin Endocrinol 2011;74:197-205.

34 Lee D-C, Lee J-W, Im J-A. Association of serum retinol binding protein 4 and insulin resistance in apparently healthy adolescents. Metabolism 2007;56:327-31.

35 Balagopal P, Graham TE, Kahn BB, et al. Reduction of elevated serum retinol binding protein in obese children by lifestyle intervention: association with subclinical inflammation. J Clin Endocrinol Metab 2007;92:1971-4

36 Kautzky-Willer A, Prager R, WaldhausI W, et al. Pronounced insulin resistance and inadequate beta-cell secretion characterize lean gestational diabetes during and after pregnancy. Diabetes Care 1997;20:1717-23.

37 Woo JG, Dolan LM, Daniels SR, et al. Adolescent sex differences in adiponectin are conditional on pubertal development and adiposity. Obes Res 2005;13:2095-101.

38 Cnop M, Havel PJ, Utzschneider KM, et al. Relationship of adiponectin to body fat distribution, insulin sensitivity and plasma lipoproteins: evidence for independent roles of age and sex. Diabetologia 2003;46:459-69.

39 Ong KK, Frystyk J, Flyvbjerg A, et al. Sex-discordant associations with adiponectin levels and lipid profiles in children. Diabetes 2006;55:1337-41.

40 Pajvani UB, Du X, Combs TP, et al. Structure-Function studies of the adipocyte-secreted hormone Acrp30/adiponectin. Implications FPR metabolic regulation and bioactivity. J Biol Chem 2003;278:9073-85.

41 Combs TP, Berg AH, Rajala MW, et al. Sexual differentiation, pregnancy, calorie restriction, and aging affect the adipocytespecific secretory protein adiponectin. Diabetes 2003;52:268-76.

$42 \mathrm{Xu} \mathrm{A}$, Chan KW, Hoo RLC, et al. Testosterone selectively reduces the high molecular weight form of adiponectin by inhibiting its secretion from adipocytes. J. Biol. Chem. 2005;280:18073-80.

43 Forest MG, De Peretti E, Bertrand J. Hypothalamic-PituitaryGonadal relationships in man from birth to puberty. Clin Endocrinol 1976;5:551-69.

44 Singh R, Artaza JN, Taylor WE, et al. Testosterone inhibits adipogenic differentiation in 3T3-L1 cells: nuclear translocation of androgen receptor complex with $\beta$-catenin and T-cell factor 4 may bypass canonical Wnt signaling to down-regulate adipogenic transcription factors. Endocrinology 2006;147:141-54.

45 Anderson LA, McTernan PG, Barnett AH, et al. The effects of androgens and estrogens on preadipocyte proliferation in human adipose tissue: influence of gender and site. J Clin Endocrinol Metab 2001;86:5045-51. 